\title{
Cash Use of the Taiwan Dollar: Is It Efficient? ${ }^{\dagger}$
}

\author{
Philip Hans Franses * $*$ and Max Welz \\ Econometric Institute, Erasmus School of Economics, P.O. Box 1738, NL3000 DR Rotterdam, The Netherlands; \\ mwelz@student.eur.nl \\ * Correspondence: franses@ese.eur.nl; Tel.: +31-104-081-273 \\ + The idea of this paper came up with the first author when he visited Taiwan in June 2018 and noticed that \\ banknotes of 200 and 2000 NT\$ did not seem to exist, while they do. \\ $\ddagger$ As of June 2018, Honorary Chair Professor, Asia University, Taichung, Taiwan.
}

Received: 21 November 2018; Accepted: 12 January 2019; Published: 15 January 2019

\begin{abstract}
Two banknotes and two coins of the New Taiwan Dollar are infrequently (if at all) used in Taiwan when people make cash payments. This note examines the effect of this behavior on the efficiency of cash payments. The results are compared with the Euro, where the two highest and two lowest tokens are also rarely used. We find for Taiwan that inefficiency increases with $60.7 \%$, while for the Euro it is only $25.3 \%$. The main reason is that two of the rarely used coins and notes in Taiwan are in the middle of the denominational range, whereas for the Euro, these tokens concern the ends of that range.
\end{abstract}

Keywords: cash payments; efficiency; denomination range; E42; E58

\section{Introduction}

Central banks issue banknotes and coins. They do so in what is called a denominational range, where usually sequences of 1, 2, and 5 are used. For example, the Euro has banknotes 5, 10, 20, 50, 100, 200, and 500. Even though many people use credit cards or debit cards when making actual payments, the use of cash is still very popular (see for example, Van der Cruijsen et al. 2017). Research on cash payments concerns the costs of cash payments (see for example, Segendorf and Jansson 2012) and how people perceive various payment modes (Khan et al. 2015). Modern payment methods developed by fintech companies also attract much research, as well as studies on the bitcoin and the like, but there is also an interest in the link between payment methods and economic development, see for example, Hasan et al. (2012). Finally, research is done on the effects of 'removing zeroes' from notes and coins in times of high inflation. In the present paper, the focus is on what happens if the paying public does not use some of the notes and coins available, where we address the case of the New Taiwan Dollar and compare it with the Euro.

The currency in Taiwan is the New Taiwan Dollar (NT\$). Its denominational range contains coins of NT\$0.50, 1, 5, 10, 20, and 50 and the banknotes NT\$100, 200, 500, 1000, and 2000. Personal observation, and also https://en.m.wikipedia.org/wiki/New_Taiwan_dollar, suggests that some coins and notes are not very popular in Taiwan. In particular the coins NT\$0.50 and 20 and the notes NT\$200 and 2000 are not often used by the Taiwanese public. In this note, we examine the consequences of these preferences for efficient payment behavior.

Efficient payment is defined as the smallest numbers of transactions involved in making a cash payment. The amount NT\$10 is efficiently paid with a coin of NT\$10. An inefficient payment for this amount would be to give a coin of NT\$20 and to get a NT\$10 coin in return. For each amount efficient payment schemes exist, and the number of efficient payments per amount can be computed. The algorithm to do this was developed in Cramer (1983), and a version has been presented in the 
Kippers et al. (2003). In this paper we provide a more complete version of the algorithm, and in Appendix A we give the relevant computer code that we used in our study.

In this note, we examine what happens with the number of efficient payments in case some notes and coins are dismissed. We first consider the case of Taiwan. Next, we compare our findings with those of the Euro. Indeed, also for the Euro there is a tendency to dismiss certain coins and notes. The Euro denominational range covers coins of $0.01,0.02,0.05,0.10,0.20,0.50,1$, and 2, while it contains the banknotes 5, 10, 20, 50, 100, 200, and 500. The 200 and 500 notes are considered to be associated with criminal activities and hence rarely visibly used. In some European countries, like in the Netherlands, all amounts are rounded at 0.05 cents, and hence the coins 0.01 and 0.02 are effectively not used. A key difference between the payment behavior for the Euro and for the NT\$ is that for the Euro it dismissed the 'end values' of the denominational range (0.01 and 0.02; 200 and 500), while in Taiwan the dismissed tokens are also midway the denominational range (like 20 and 200). We document that $60.7 \%$ more notes and coins are required to make efficient payments in Taiwan, while for the Euro this is just $25.3 \%$. The dismissed coins and notes in Taiwan thus lead to substantial payment inefficiency.

\section{Method}

Denote with $A$ the payment amount and with $N(A)$ the amount of tokens (notes and or coins) to be used for cash payment. The denomination range is given by the set $\{1,2, \ldots, D\}$ and an element of this set is denoted by $d$. For Taiwan, this set is NT\$0.50, 1, 5, 10, 20, and 50 for coins, and the country has the following banknotes, that is, NT\$100, 200, 500, 1000, and 2000.

Denote with $N(A, d)$ the number of tokens of denomination $d$, so

$$
N(A)=\sum_{d=1}^{D} N(A, d)
$$

Finally, denote $v(d)$ as the value of denomination $d$.

The objective is now to minimize $n(A)$ by choosing the proper notes and coins out of the set $\{1,2, \ldots, D\}$. More formally, one should solve

$$
\begin{aligned}
& \text { minimize } n^{*}(A)=\sum_{d=1}^{D}|n(A, d)| \\
& \text { subject to } \sum_{d=1}^{D} n(A, d) v(d)=A
\end{aligned}
$$

Note that we consider the absolute value of $n(A, d)$ to allow for the possibility that notes and coins are returned as change.

The algorithm of Cramer to obtain all efficient payment schemes proceeds as follows. We take a range of payment amounts that are of potential interest, say NT $\$ 0.50$ until NT $\$ 35,700$, with intervals of NT $\$ 0.50$. We choose for NT $\$ 35,700$ as with the current exchange rate (November 2018) this amount is about equal to 1000 Euro. The algorithm seeks to find for each of these amounts the efficient combinations of notes and coins. A general pseudocode implementation of this algorithm can be found in Appendix A.

Step 1: The algorithm starts by taking all amounts that can be paid with only one note or one coin. For the New Taiwan Dollar, the amounts NT\$0.50, 1, 5, 10, 20, 50, 100, 200, 500, 1000, and 2000 are now covered.

Step 2: All amounts that can be paid with two tokens, either two given by the individual as payment or one returned by someone else as change, are next to be computed. For example, NT\$150 can be paid efficiently with notes of NT $\$ 100$ and 50, but also with NT $\$ 200$ and 50 as change. All such combinations that constitute an efficient payment for a given amount are stored. If we now observe an 
amount that was already covered with only one token in the previous step, we do not add this pair of two tokens to the list as these are obviously not efficient for this amount.

Step 3: To the pairs that were found efficient in the previous two steps, we add each token once, both with positive and negative sign, the latter indicating change. For example, to the NT\$1000 and 500 notes we add a NT\$20 coin, covering the amounts 1520 and 1480 (where in the last case the NT\$20 is given as change). For a given pair in Step 2, this gives $2 D$ extra combinations with an additional token each. Adding a token with a positive sign to a combination which has this same token with a negative sign (and vice versa) would yield a combination with less tokens and is therefore ignored.

Note that there are two restrictions which need to be taken into account, and it is here where the method of Kippers et al. (2003) is modified. First, we do not consider combinations which lead to infeasible amounts, that is, amounts that are lower or higher than the lowest or highest amounts that we consider. In our case, that would be amounts that are lower than NT $\$ 0.50$ and higher than NT $\$ 35,700$.

Second, duplicates may occur in this step. In different stages of this step, we may obtain the exact same combination multiple times, so we also do not consider such duplicates. Hence, each efficient payment scheme is unique.

Step 4: Repeat Step 3 by increasing each time the number of tokens until all amounts between NT $\$ 0.50$ until NT $\$ 35,700$ are covered.

When all tokens are considered the payment amount ranges from NT\$0.50 to NT\$35,700 with steps of NT $\$ 0.50$. However, when tokens NT $\$ 0.50,20,200$, and 2000 are excluded, the payment amount ranges from NT\$1 to NT $\$ 35,700$ with steps of NT $\$ 1$.

A similar exercise is carried out for the Euro, now with amounts 0.01 to 1000 Euros. For the Euro we also consider the cases where the 200 and 500 Euro notes are not used, and where additionally the 0.01 and 0.02 Euro cents are dismissed.

\section{Results}

For Taiwan, the results of our exercise appear in Table 1. If all tokens are considered for all payment amounts until and including NT\$35,700, we see that there are 195,659 efficient payment schemes. The average amount of tokens exchanged is 14.030 , where the maximum amount is 25 . When the tokens NT\$0.5, 20, 200, and 2000 are excluded, we see that the number of efficient schemes reduces, but this is also due to the fact all amounts are now rounded at NT\$1. A more important figure is the new average amount of tokens exchanged, which becomes 22.546. This entails an increase of inefficiency of $60.7 \%$. The maximum number even increases to 43 .

Table 1. Results for New Taiwan Dollar on efficient payment schemes (percentage increase, relative to the all tokens case) in parentheses.

\begin{tabular}{ccc}
\hline Statistic & All Tokens & Excluded Tokens Are NT\$0.50, 20, 200, and 2000 \\
\hline $\begin{array}{c}\text { Amount of efficient schemes } \\
\text { Amount of tokens }\end{array}$ & 195,659 & 47,073 \\
exchanged & & \\
Average & 14.030 & $22.546(60.7 \%)$ \\
Median & 13 & $22(69.2 \%)$ \\
Minimum & 1 & 1 \\
Maximum & 25 & $43(72.0 \%)$ \\
\hline
\end{tabular}

Note: when all tokens are considered the payment amount ranges from NT\$0.5 to NT\$35,700 with steps of NT\$0.5. When tokens are excluded the payment amount ranges from NT $\$ 1$ to NT $\$ 35,700$ with steps of NT $\$ 1$.

To put these numbers in perspective, consider the results for the Euro in Table 2. For the range of 0.01 Euro to 1000 Euros, there are 577,066 efficient payment schemes. The average amount of tokens exchanged is 7.534. When we delete the 200 and 500 Euro notes, this average increases to 10.333 (a $37.2 \%$ increases of inefficiency), where when additionally the 0.01 and 0.02 cents are dismissed, the 
average of exchanged tokens across efficient payment schemes increases to 9.429. This latter increase of inefficiency is $25.3 \%$.

Table 2. Results for Euro on efficient payment schemes (percentage increase, relative to the all tokens case) in parentheses.

\begin{tabular}{cccc}
\hline Statistic & All Tokens & $\begin{array}{c}\text { Excluded Tokens Are } \\
\text { 200 and 500 Euro }\end{array}$ & $\begin{array}{c}\text { Excluded Tokens Are 0.01, 0.02, } \\
\text { 200, and 500 Euro }\end{array}$ \\
\hline $\begin{array}{c}\text { Amount of efficient schemes } \\
\text { Amount of tokens }\end{array}$ & 577,066 & 365,910 & \\
exchanged & & & \\
Average & 7.534 & $10.333(37.2 \%)$ & $9.429(25.3 \%)$ \\
Median & 6 & $10(66.7 \%)$ & $9(50 \%)$ \\
Minimum & 1 & 1 & 1 \\
Maximum & 11 & $17(54.5 \%)$ & $16(45.5 \%)$ \\
\hline
\end{tabular}

Note: when all tokens are considered the payment amount ranges from 0.01 Euro to 1000 Euro with steps of 0.01 Euro. This also holds for the case where 200 and 500 Euro notes are dismissed. When additionally the 0.01 and 0.02 Euro cents are excluded the payment amount ranges from 0.05 Euro to 1000 Euro with steps of 0.05 Euro.

\section{Conclusions}

The overall conclusion of our exercise in this note is that excluding NT\$0.50, 20, 200, and 2000 in Taiwan leads to a marked inefficiency in payment behavior, much more than the dismissal of Euro coins and notes implies. The main reason is that NT\$20 and 200 are in the middle of the denominational range, whereas for the Euro the dismissed tokens appear at the end of the range.

Our exercise is a pure theoretical exercise, and it remains to be seen what happens when people make actual payments. Such empirical work involves tedious data collection, as is reported in Franses and Kippers (2007). The main reason is that the data collectors should also observe the wallet content of individuals, in order to see amongst which coins and notes the individuals can choose. In Franses and Kippers (2010), this tedious data collection is somewhat alleviated by making people play Monopoly games, where the wallet contents can be observed on the table. Further empirical research in Taiwan can lead to interesting insights.

Author Contributions: Conceptualization, P.H.F.; methodology, P.H.F. and M.W.; software, M.W.; writing—original draft preparation, P.H.F.; writing—review and editing, P.H.F. and M.W.; supervision, P.H.F.

Funding: This research received no external funding.

Acknowledgments: We thank two anonymous reviewers for helpful suggestions.

Conflicts of Interest: The author declares no conflicts of interest.

\section{Appendix A Computer Code}

We have listed the general program we wrote for our analysis as pseudocode here. The original MATLAB program can be provided on request, please contact the first author for such requests. 


\begin{abstract}
\# Initialize
D_set, A_set are column vectors that hold the currency denominations and amounts to be taken into account, respectively. P_mat is a three-dimensional array of zeros that will hold the efficient payment schemes in its columns. Each row corresponds to one token (from D_set, in that order), so each cell is a token counter, where negative numbers are change. Only the number of non-zero columns will correspond to the number of efficient payment schemes for a given amount.
\end{abstract}

\#\# Step 1:

for every amount in A_set:

Cover all amounts that can be covered with only one token. Always start to fill the columns end(for) in the corresponding first column.

\#\# Step 2:

for every amount in A_set:

if the first column of this iteration's contains nonzero elements: continue;

end(if)

for $i, j$ in D_set:

\# All tokens given by customer:

if (D_set[i] + D_set[j] == amount):

Increment corresponding token counters of this amount's matrix. end(if)

\# Situation with change:

if (amount - D_set[i]) == D_set[j] * $(-1)$

Increment or decrement (change) corresponding token counters of this

end(if)

end(for)

end(for)

Replace all duplicate columns by zero columns.

\#\# Steps 3 and 4 :

prv_tokens = 2; \# number of tokens used in previous step, so 2 here.

while there is a first column in P_mat that holds only zeros:

for every amount in A_set:

if there was no efficient number of tokens found in previous step: continue;

end(if)

add_token_mat = zeros(D, 2D); \# to hold the additional tokens

for every column that holds prv_tokens in P_mat:

Add each token once, both with positive and negative sign to add_token_mat. Make sure to consider only valid columns of add_token_mat from here on (valid columns are columns that do not lead to an amount that is either lower or higher than the minimum or maximum, respectively, amount we consider).

Find the corresponding amount of each valid column and add this column

end(for) to that amount's matrix in P_mat provided that it is not already there.

end(for)

Update prv_tokens;

end(while) 
Every nonempty column in P_mat will be unique and correspond to one efficient payment scheme.

\section{References}

Cramer, Jan S. 1983. Currency by denomination. Economics Letters 12: 299-303. [CrossRef]

Kippers, Jeanine, Erjen van Nierop, Richard Paap, and Philip Hans Franses. 2003. An empirical study of cash payments. Statistica Neerlandica 57: 484-508. [CrossRef]

Franses, Philip Hans, and Jeanine Kippers. 2007. An empirical analysis of euro cash payments. European Economic Review 51: 1985-97. [CrossRef]

Franses, Philip Hans, and Jeanine Kippers. 2010. How do we pay with euro notes when some notes are missing? Empirical evidence from Monopoly experiments. Applied Financial Economics 20: 459-64. [CrossRef]

Hasan, Iftekhar, Heiko Schmiedel, and Liang Song. 2012. Return from retail banking and payments. Journal of Financial Services Research 41: 163-95. [CrossRef]

Khan, Jashim, Russell W. Belk, and Margaret Craig-Lees. 2015. Measuring consumer perceptions of payment mode. Journal of Economic Psychology 47: 34-49. [CrossRef]

Segendorf, Bjorn, and Thomas Jansson. 2012. The Cost of Consumer Payments in Sweden. Sveriges Riksbank Working Paper No. 262. Stockholm: Sveriges Riksbank.

Van der Cruijsen, Carin, Lola Hernandez, and Nicole Jonker. 2017. In love with the debit card but still married to cash. Applied Economics 49: 2989-3004. [CrossRef]

(C) 2019 by the authors. Licensee MDPI, Basel, Switzerland. This article is an open access article distributed under the terms and conditions of the Creative Commons Attribution (CC BY) license (http://creativecommons.org/licenses/by/4.0/). 